\title{
A Proteomic View to Characterize the Effect of Chitosan Nanoparticle to Hepatic Cells: Is Chitosan Nanoparticle an Enhancer of PI3K/AKT1/mTOR Pathway?
}

\author{
Ming-Hui Yang, ${ }^{1,2,3}$ Shyng-Shiou Yuan, ${ }^{2,3,4,5}$ Ying-Fong Huang, ${ }^{6,7}$ Po-Chiao Lin, \\ Chi-Yu Lu, ${ }^{9,10}$ Tze-Wen Chung, ${ }^{11}$ and Yu-Chang Tyan ${ }^{2,6,9,12}$ \\ ${ }^{1}$ Instrument Technology Research Center, National Applied Research Laboratories, Hsinchu 300, Taiwan \\ ${ }^{2}$ Translational Research Center, Kaohsiung Medical University Chung-Ho Memorial Hospital, Kaohsiung 807, Taiwan \\ ${ }^{3}$ Department of Medical Research, Kaohsiung Medical University Chung-Ho Memorial Hospital, Kaohsiung 807, Taiwan \\ ${ }^{4}$ Department of Obstetrics and Gynecology, Kaohsiung Medical University Chung-Ho Memorial Hospital, Kaohsiung 807, Taiwan \\ ${ }^{5}$ School of Medicine, College of Medicine, Kaohsiung Medical University, Kaohsiung 807, Taiwan \\ ${ }^{6}$ Department of Medical Imaging and Radiological Sciences, Kaohsiung Medical University, Kaohsiung 807, Taiwan \\ ${ }^{7}$ Department of Nuclear Medicine, Kaohsiung Medical University Chung-Ho Memorial Hospital, Kaohsiung 807, Taiwan \\ ${ }^{8}$ Department of Chemistry, National Sun Yat-sen University, Kaohsiung 804, Taiwan \\ ${ }^{9}$ National Sun Yat-sen University-Kaohsiung Medical University Joint Research Center, Kaohsiung 807, Taiwan \\ ${ }^{10}$ Department of Biochemistry, College of Medicine, Kaohsiung Medical University, Kaohsiung 807, Taiwan \\ ${ }^{11}$ Department of Biomedical Engineering, National Yang-Ming University, Taipei 112, Taiwan \\ ${ }^{12}$ Center of Biomedical Engineering and System Biology, Kaohsiung Medical University, Kaohsiung 807, Taiwan
}

Correspondence should be addressed to Tze-Wen Chung; twchung@ms.ym.edu.tw and Yu-Chang Tyan; yctyan@kmu.edu.tw

Received 27 November 2013; Accepted 10 February 2014; Published 16 March 2014

Academic Editor: Yoshihiko Hayashi

Copyright (c) 2014 Ming-Hui Yang et al. This is an open access article distributed under the Creative Commons Attribution License, which permits unrestricted use, distribution, and reproduction in any medium, provided the original work is properly cited.

Chitosan nanoparticle, a biocompatible material, was used as a potential drug delivery system widely. Our current investigation studies were the bioeffects of the chitosan nanoparticle uptake by liver cells. In this experiment, the characterizations of chitosan nanoparticles were measured by transmission electron microscopy and particle size analyzer. The average size of the chitosan nanoparticle was $224.6 \pm 11.2 \mathrm{~nm}$, and the average zeta potential was $+14.08 \pm 0.7 \mathrm{mV}$. Moreover, using proteomic approaches to analyze the differential protein expression patterns resulted from the chitosan nanoparticle uptaken by HepG2 and CCL-13 cells identified several proteins involved in the PI3K/AKT1/mTOR pathway. Our experimental results have demonstrated that the chitosan nanoparticle may involve in the liver cancer cell metastasis and proliferation.

\section{Introduction}

Biomaterials play important roles in regenerative medicine, tissue engineering, and drug delivery [1]. Nanomedicine is the application of nanotechnology in medicine, which enabled the development of nanoparticle therapeutic carriers. These drug carriers are targeted to tumor cell surfaces through the enhanced permeability and retention effect; thus, they are very suitable for the chemotherapeutics delivery in cancer treatment. Nanomaterials have increased surface to volume ratio compared with their bulk materials, and this may confer interesting properties, such as increased mechanical strength. Their distinct physicochemical characteristics, obtained by changing the size and shape, are very different from their natural materials and thus granting new possibilities [2]. Different nanomaterials have various effects on cells. For example, the uptake of silver nanoparticles caused cell proliferation inhibition in mouse leukaemic monocyte macrophage cells [3] and human keratinocytes [4]. In addition, low concentration of gold nanoparticles 
resulted reduced cell proliferation in rat pheochromocytoma cells and human umbilical vein endothelial cells [5]. On the other hand, single-wall carbon nanotubes (SWCNTs) were investigated for biomedical applications and showed no negative effect for cell proliferation [6].

Chitosan, a biocompatible and biodegradable polymer, is a modified natural carbohydrate polymer prepared by the partial N-deacetylation of chitin (primary unit: 2-deoxy-2(acetylamino) glucose). Chitosan and chitin, next to cellulose, are the second most plentiful nature and nontoxic, biodegradable cationic polymers. It is a natural biopolymer derived from crustacean shells such as krill, shrimps, lobsters, and crabs [1]. As such, chitosan is an abundant natural polymer available from a renewable resource. Chitosan, a mucopolysaccharide having structural characteristics similar to glycosamines, is a linear $\beta(1 \rightarrow 4)$-D-glucosamine and acetyl- $\beta(1 \rightarrow 4)$-D-glucosamine, which can be obtained by alkaline $\mathrm{N}$-deacetylation derivative of chitin [7]. Thus, chitosan is usually not a homopolymer of D-glucosamine but a copolymer containing less than $40 \% \mathrm{~N}$-acetyl-Dglucosamine residues. Chitosan has both reactive amino and hydroxyl groups, which can be used to chemically alter these properties under mild reaction conditions. Therefore, there are many interesting chitosan derivatives, especially for biomedical applications $[1,8,9]$. Chitosan has been proposed for the development of membranes and fibers of hemodialysis and blood oxygenators, skin substitute, wound dressing and suture materials intended for immobilization of enzymes and cells to bind with bile and fatty acids and as a vehicle for drug and gene delivery [10-14]. It has been widely used in several fields of developing treatments as diverse as lung surfactant additives, wound healing, and tissue engineering. Although chitosan is suitable for medical applications, for those applications that involve blood contact such as hemodialysis membranes, chitosan promotes surface induced thrombosis and embolization [10, 12]. It is indicated in the literature that chitosan has the capacity to activate both complement and blood coagulation system $[15,16]$. Chitosan is also a bioactive carbohydrate polymer and potentially useful in tissue engineering and for gene and drug delivery [17, 18]. However, none is without disadvantages. For example, chitosan may prevent the absorption of needed vitamins and minerals. It may also be dangerous to those who are allergic to shellfish.

Chitosan nanoparticle prepared by ionotropic gelation technique was first reported by Calvo et al. [19]. It can be formed with sodium tripolyphosphate. The chitosan nanoparticles have gained more attention as drug delivery carriers because of better stability, low toxicity, simple and mild preparation method, and providing versatile routes of administration [20]. Chitosan nanoparticles accumulation typically occurs around the defect area in cells and tissues by hydrophobic interactions. In addition, several physiochemical characteristics of chitosan nanoparticles, such as abilities to cross biological barriers, to protect macromolecules from degradation and to deliver a compound at a target site, were examined as favorable [21].

The development of nanoparticulate drug carriers has followed several routes depending on the final application.
Although a wide variety of systems have been designed with their own advantages and limitations, the common goal is to rationalize drug delivery to enhance the bioavailability of the drugs towards targeted diseased cells, promoting the required response while minimizing side effects. Therefore, to use chitosan derivatives for biomedical applications, a test for evaluating biocompatibility must be performed.

In this study, we investigated various methods to analyze and characterize the parameters that influence the uptake of cells on chitosan nanoparticle. The CCL-13 and HepG2 cells were served as cell models for the uptake of chitosan nanoparticles. For instance, using colorimetric techniques such as LDH or BrdU assay is a convenient method and typically applied to many biomaterial researches. Characterizations of chitosan nanoparticles were observed by TEM, particle sizer, and zeta potential. To evaluate early responses of CCL-13 and HepG2 cells to chitosan nanoparticles, a mass spectrometry-based profiling system was adopted for assessing characteristic proteins that were expressed due to the interactions of CCL-13 and HepG2 cells with chitosan nanoparticles. Through the investigation, various proteins that influence the early responses of CCL-13 and HepG2 cells on chitosan nanoparticle were found, and, as we know, some of them have not been reported in the study of cellnanoparticle interactions.

\section{Materials and Methods}

2.1. Chitosan Nanoparticle Preparation. Chitosan nanoparticles were prepared according to Calvo et al. [19]. Briefly, water-soluble chitosan was dissolved in aqueous solution. Nanoparticles were formed spontaneously upon addition of $2 \mathrm{~mL}$ of the sodium tripolyphosphate (TPP in sodium citrate, 238503, Sigma-Aldrich) aqueous solution (10\%) to $100 \mathrm{~mL}$ of the chitosan acidic solution (448869, Sigma-Aldrich, low molecular weight, $75-85 \%$ deacetylation, $1 \mathrm{mg} / \mathrm{mL}$, w/v) under magnetic stirring at room temperature. The $\mathrm{pH}$ of chitosan solution varied between 3.0 and 5.0. PEG $(10 \mathrm{mg} / \mathrm{mL}$, $1 \mathrm{~mL}$ ) was dissolved in the chitosan solution after the addition of the TPP solution. Nanoparticles were isolated by ultracentrifugation $(25,000 \mathrm{~g}, 15 \mathrm{~min})$ and then resuspended in water by manual shaking.

2.2. Characterization of Chitosan Nanoparticles. The morphological examination of the chitosan nanoparticles was performed by transmission electron microscopy at an accelerating voltage of $100 \mathrm{kV}$ (TEM, JEM 1200CX II, JEOL). The sample was stained with $2 \%(\mathrm{w} / \mathrm{v})$ phosphotungstic acid and placed on copper grids for viewing by TEM.

The particle size and size distributions of the chitosan nanoparticles were performed by particle size analyzer ( 90 plus particle sizer, Brookhaven Instruments Corp., USA). For the particle size analysis, each sample was diluted to the appropriate concentrating with filtered distilled water. Each analysis lasted $2 \mathrm{~min}$ and was performed at $25^{\circ} \mathrm{C}$ with an angle detection of $90^{\circ}$. 
Measurement of the zeta potential of nanoparticles was performed by Zeta plus 90 particle sizer (Brookhaven Instruments Corp., USA) with a $5 \mathrm{~mW} \mathrm{He-Ne} \mathrm{laser}(\lambda=$ $663 \mathrm{~nm}$ ). The zeta potential values were calculated from the mean electrophoretic mobility values using Smoluchowski's equation.

2.3. Cell Culture. HepG2 (liver tumor cell) and CCL-13 (liver normal cell) cells were maintained at $37^{\circ} \mathrm{C}$ and $5 \% \mathrm{CO}_{2}$ in RPMI 1640 medium (Gibco, USA) supplemented with 10\% fetal bovine serum (FBS, Hyclone Laboratories, Logan, UT), $1 \%$ penicillin/streptomycin (Gibco, Grand Island, NY, USA), and $44 \mathrm{mM} \mathrm{NaHCO}$ (Sigma, USA). After three days, the cells were washed with serum-free RPMI 1640 medium and incubated with the serum-free medium containing chitosan nanoparticles at concentrations of 1 to $5 \mu \mathrm{g} / \mathrm{mL}$ for $12 \mathrm{~h}$.

2.4. BrdU and LDH Assay. CCL-13 and HepG2 cells were seeded in a sterile 96-well tissue culture plate at $2 \times$ $10^{5}$ cells $/ \mathrm{mL}$ in $100 \mu \mathrm{L} /$ well of appropriate cell culture media with chitosan nanoparticles. The cell proliferation was determined by bromodeoxyuridine assay (BrdU Cell Proliferation Assay, Millipore, USA). The cytotoxicity of chitosan nanoparticles was evaluated in vitro using the lactate dehydrogenase assay (LDH Cytotoxicity Assay, ScienCell Research Laboratories, USA). These assays were performed according to the manufacturers' instructions. The absorbance values were measured by an ELISA reader (Multiskan EX, Thermo scientific, Vantaa, Finland, reference wavelength: $450 \mathrm{~nm}$ ).

2.5. Cell Morphology. For cell morphologies of HepG2 and CCL-13 before and after incubation with chitosan nanoparticles, the cell live images were observed with a microscope equipped with fluorescence light source (FLoid cell fluorescence imaging Station, Invitrogen), and the cell micrographs were taken with a CCD camera.

2.6. Protein Sample Preparation. After incubation with chitosan nanoparticles, the HepG2 and CCL-13 cells were lysed by cell lysis buffer (3500-1, Epitomics, Inc, USA), and cell lysates were centrifuged at $1500 \times \mathrm{g}$ for $10 \mathrm{~min}$ at $4^{\circ} \mathrm{C}$. The supernatants were flitted by $0.8 \mu \mathrm{m}$ filter and the protein concentrations were adjusted to $1 \mathrm{mg} / \mathrm{mL}$ by $25 \mathrm{mM}$ ammonium bicarbonate.

Cell lysates samples $(100 \mu \mathrm{L})$ were transferred into the $1.5 \mathrm{~mL}$ Eppendorf tubes and incubated at $37^{\circ} \mathrm{C}$ for $3 \mathrm{~h}$ after mixing with $25 \mu \mathrm{L}$ of $1 \mathrm{M}$ dithiothreitol (DTT, USB Corporation, 15397). Then cell lysates samples were reduced and alkylated in the dark at room temperature for $30 \mathrm{~min}$ after addition of $25 \mu \mathrm{L}$ of $1 \mathrm{M}$ iodoacetamide (IAA, Amersham Biosciences, RPN6302V) in $25 \mathrm{mM}$ ammonium bicarbonate. Approximately $10 \mu \mathrm{L}$ of $0.1 \mu \mathrm{g} / \mu \mathrm{L}$ modified trypsin digestion buffer (Trypsin Gold, Mass Spectrometry Grade, V5280, Promega, WI, USA) in $25 \mathrm{mM}$ ammonium bicarbonate was added to the cell lysates samples, and the cell lysates samples were incubated at $37^{\circ} \mathrm{C}$ for at least $12 \mathrm{~h}$ in a water bath. Two microliter of formic acid was added to each sample before mass spectrometric analysis for protein identification.
2.7. Proteomic Analysis. The complex peptide mixtures were separated by RP-nano-UPLC-ESI-MS/MS. The protein tryptic digests were fractionated using a flow rate of $400 \mathrm{~nL} / \mathrm{min}$ with a nano-UPLC system (nanoACQUITY UPLC, Waters, Milford, MA) coupled to an ion trap mass spectrometer (LTQ Orbitrap Discovery Hybrid FTMS, Thermo, San Jose, $\mathrm{CA})$ equipped with an electrospray ionization source. For RP-nano-UPLC-ESI-MS/MS, a sample $(2 \mu \mathrm{L})$ of the desired peptide digest was loaded into the reverse phase column (Symmetry C18, $5 \mu \mathrm{m}, 180 \mu \mathrm{m} \times 20 \mathrm{~mm}$ ) by an autosampler. The RP separation was performed using a linear acetonitrile gradient from $99 \%$ buffer A (100\% D.I. water/0.1\% formic acid) to $85 \%$ buffer B (100\% acetonitrile/ $0.1 \%$ formic acid) in $100 \mathrm{~min}$ using the micropump. The separation is performed on a C18 microcapillary column (BEH C18, $1.7 \mu \mathrm{m}, 75 \mu \mathrm{m} \times$ $100 \mathrm{~mm}$ ) using the nanoseparation system. As peptides eluted from the microcapillary column, they were electrosprayed into the ESI MS/MS with the application of a distal $2.1 \mathrm{kV}$ spraying voltage with heated capillary temperature of $200^{\circ} \mathrm{C}$. Each cycle of one full scan mass spectrum (m/z 400-2000) was followed by four data dependent tandem mass spectra with the collision energy set at 35\%.

2.8. Database Search. For protein identification, Mascot software (Version 2.2.1, Matrix Science, London, UK) was used to search the Swiss-Prot human protein sequence database. Positive protein identifications were defined when Mowse scores greater than 100 were considered significant $(P<$ 0.05). Proteins were annotated by similar searches using UniProtKB/Swiss-Prot databases. The protein-protein interaction pathways were performed by String $9.1 \mathrm{Web}$ software.

2.9. Statistical Analysis. All calculations used the SigmaStat statistical software (Jandel Science Corp., San Rafael, CA). All statistical significances were evaluated at $95 \%$ of confidence level or better. Data are presented as mean \pm standard error.

\section{Results and Discussions}

3.1. Size, Zeta Potential, and Morphology of Chitosan Nanoparticles. As determined by particle size and zeta potential analyzers, the average size of the chitosan nanoparticle was $224.6 \pm 11.2 \mathrm{~nm}$, and the average zeta potential was $+14.08 \pm$ $0.7 \mathrm{mV}$ in phosphate-buffered saline. The size and surface morphology of chitosan nanoparticles was shown in Figure 1. The TEM image displays the clear spherical morphology of the chitosan nanoparticles having a mean diameter of chitosan nanoparticles about $236.3 \mathrm{~nm}$ as shown. Zeta potential is the surface charge of nanoparticles and can influence the nanoparticle stability in suspension through the electrostatic repulsion between nanoparticles. In this study, the surface charge of chitosan nanoparticles was positive, according to the protonation of $\mathrm{NH}_{2}$ functional groups of glucosamine units to $\mathrm{NH}_{3}{ }^{+}$ion.

3.2. In Vivo Chitosan Nanoparticle Uptake. In vitro uptake of chitosan nanoparticles was evaluated by fluorescence microscopy. CCL-13 and HepG2 cells were incubated with 


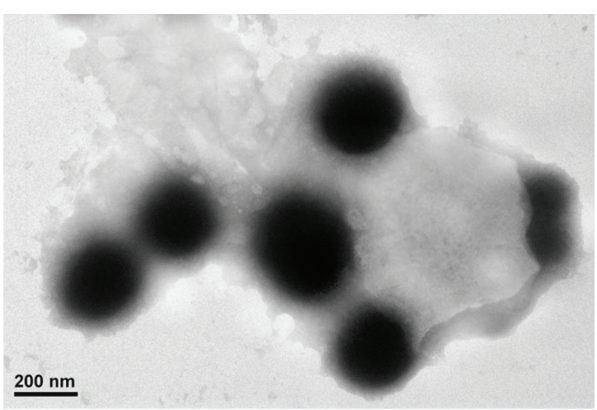

(a)

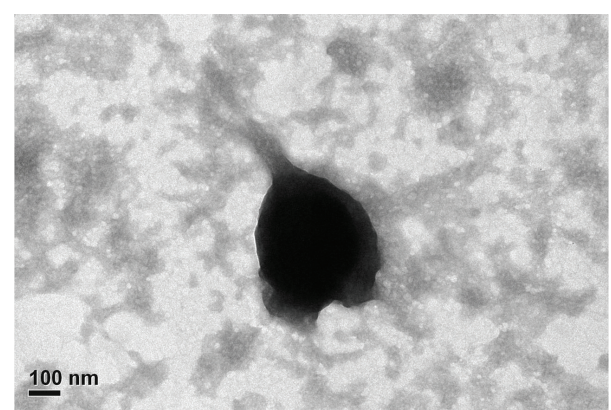

(b)

FIGURE 1: The morphological examination of the chitosan nanoparticles was performed by transmission electron microscopy at an accelerating voltage of $100 \mathrm{kV}$. The mean of diameter is around $236.3 \mathrm{~nm}$.

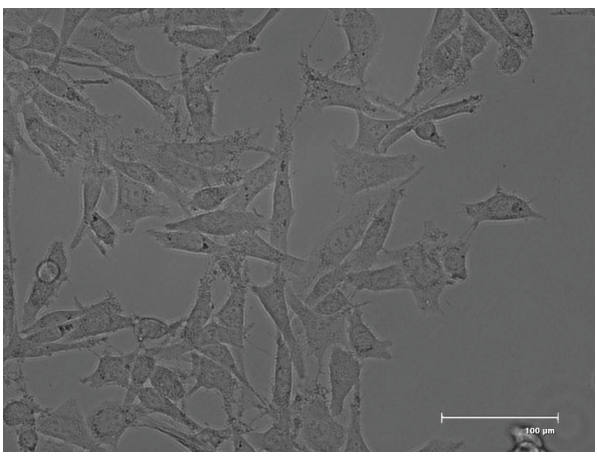

(a)

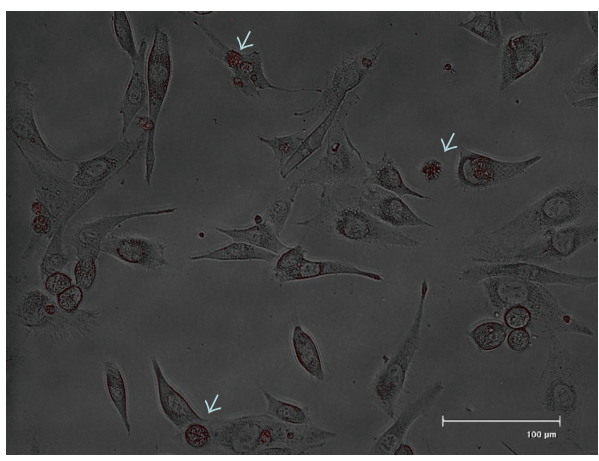

(c)

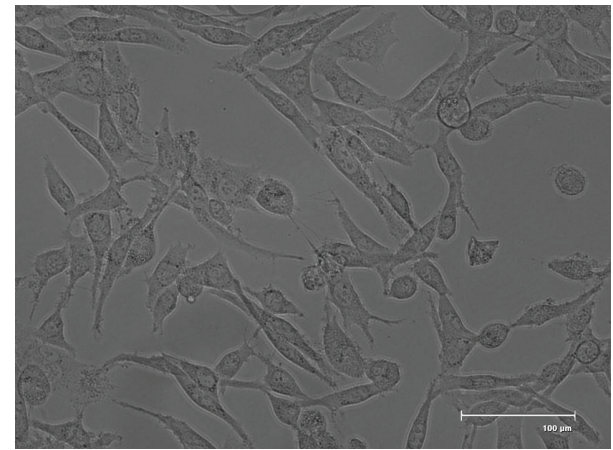

(b)

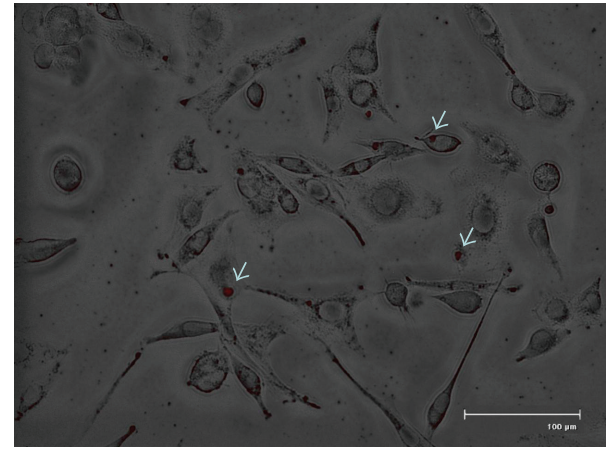

(d)

Figure 2: The live fluorescent images of chitosan nanoparticles (red) taken by CCL-13 cells (a, c) and HepG2 cells (b, d). (a) and (b): cells without chitosan nanoparticles; (c) and (d): cells with chitosan nanoparticles at a concentration of $5 \mu \mathrm{g} / \mathrm{mL}$ for $12 \mathrm{~h}$ at $37^{\circ} \mathrm{C}$; the red fluorescence was localized near by the cell nuclei. Those images represented merged images of DIC and red fluorescence. (600x, scale bar: $100 \mu \mathrm{m})$.

the growth medium containing chitosan nanoparticles at a concentration of $5 \mu \mathrm{g} / \mathrm{mL}$ for $12 \mathrm{~h}$ at $37^{\circ} \mathrm{C}$, respectively. As expected, no red fluorescence signals were detected in sections of the cells without chitosan nanoparticles (Figure 2(a): CCL-13 and Figure 2(b): HepG2, 600x, scale bar: $100 \mu \mathrm{m}$ ). Cell micrographs from chitosan nanoparticles treated CCL13 and HepG2 cells reveled red fluorescence localized near by the cell nuclei (Figure 2(c): CCL-13 and Figure 2(d): HepG2, 600x, scale bar: $100 \mu \mathrm{m})$. In some HepG2 cells, the fluorescence was also localized in the cytoplasm.

3.3. Cytotoxicity of Chitosan Nanoparticle. To examine the cytotoxicity, HepG2 and CCL-13 cells were incubated with chitosan nanoparticles for $12 \mathrm{~h}$. LDH and BrdU assays are quantitative colorimetric assays for mammalian cell survival and cell proliferation.

The cell death is assayed by the quantification of a stable cytoplasmic enzyme activity, LDH, which was released into the cell culture supernatant upon cell death and damage of the cytoplasmic membrane. BrdU is integrated into newly synthesized DNA strands of actively proliferating cells. Following partial denaturation of double stranded DNA, BrdU is detected immunochemically allowing the assessment of the number of cells which are synthesizing DNA. As shown in Figure 3, the LDH concentrations were decreased and observed between the groups treated with different concentration of chitosan nanoparticle and control 

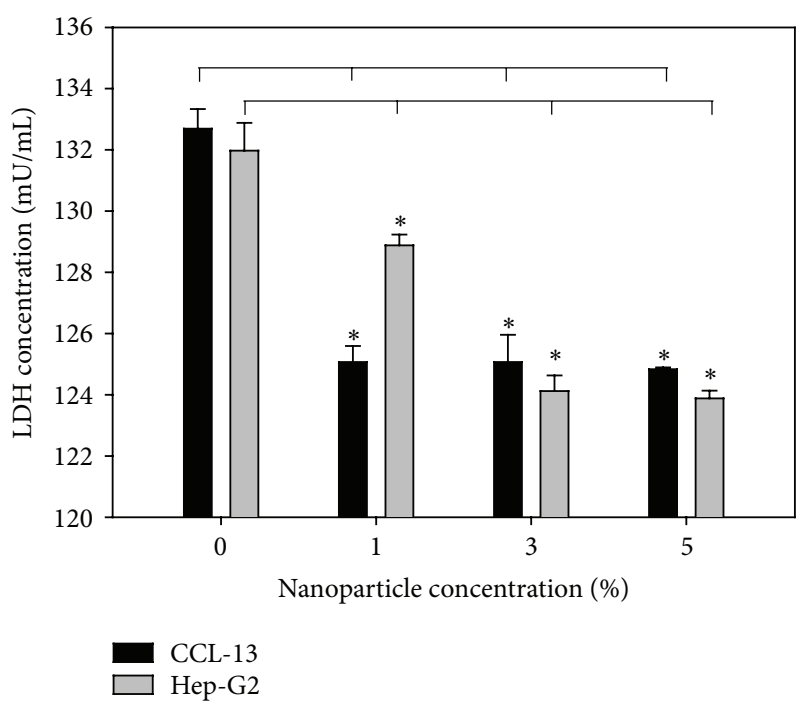

Figure 3: LDH test of chitosan nanoparticle (CSNP) effects on Chang and HepG2 cells $(N=6$, mean \pm standard error, $t$-test, $P<0.05)$.

$(P<0.05, N=6)$. Compared with the control, the BrdU was upregulated in the groups treated with chitosan nanoparticle with significantly increase (Figure $4, P<0.05, N=6$ ). Those results indicate that the chitosan nanoparticle was no significant cytotoxicity observed; in addition, the chitosan nanoparticle improved the cell growth and proliferation. In previous studies, it was indicated that the metal or metal oxide nanoparticles were with high cell toxicity [22-24]. Unlike metal or metal oxide nanoparticles, the chitosan nanoparticle was nontoxic. The cell growth and survival rate were increased with the higher concentration of chitosan nanoparticles. As shown in Figures 3 and 4, the chitosan nanoparticle was enhanced cell growth in a dose dependent manner.

3.4. Proteomic Analysis of Cell Response to Chitosan Nanoparticle. To investigate the effect of chitosan nanoparticle on liver normal and tumor cells, a proteomic approach, such as RP-nano-UPLC-ESI-MS/MS analysis, was utilized to analyze cell lysates. The traditional methods use individual antibodies to evaluate the cell response to nanoparticles, but the proteomic approach can be used to analyze an enormous number of proteins simultaneously. In this study, HepG2 and CCL-13 cells were incubated with chitosan nanoparticles. After $12 \mathrm{~h}$, the HepG2 and CCL-13 cells were lysed, and the cell lysates were digested by trypsin; generating tryptic peptides was subsequently analyzed by RP-nano-HPLC-ESI-MS/MS, respectively. The RP-nano-HPLC-ESI-MS/MS approach is perhaps the most representative method in proteome researches. The fragmentation spectra obtained by the RP-nano-HPLC-ESIMS/MS analysis in gradient detection mode were compared with a nonredundant protein database using Mascot software. All Mascot results were visually confirmed. In addition, the criterion requires a readily observable series of at least four y ions for an identified peptide [25]. When a protein

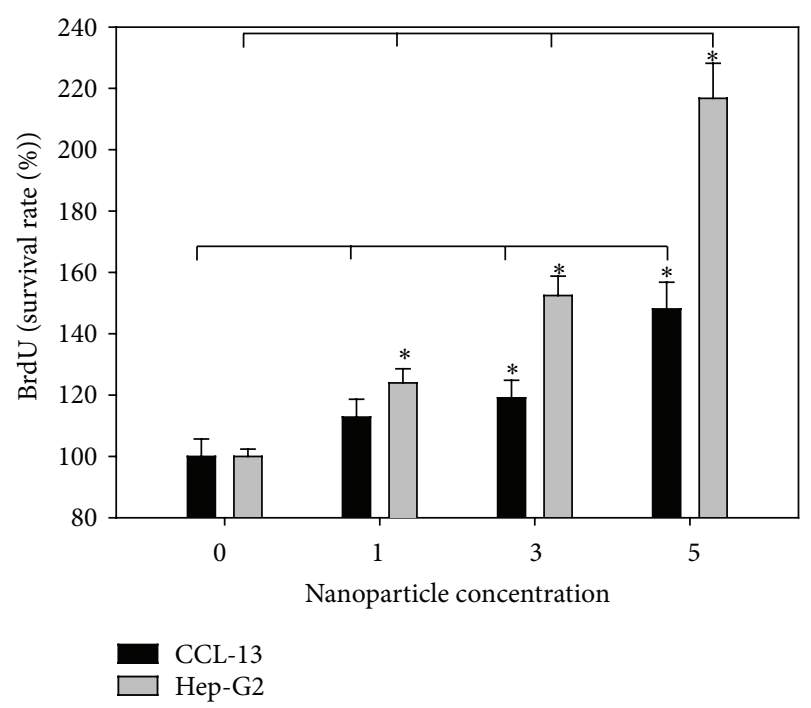

Figure 4: Proliferation (BrdU) test of chitosan nanoparticle (CSNP) effects on Chang and HepG2 cells $(N=6$, mean \pm standard error, t-test, $P<0.05)$.

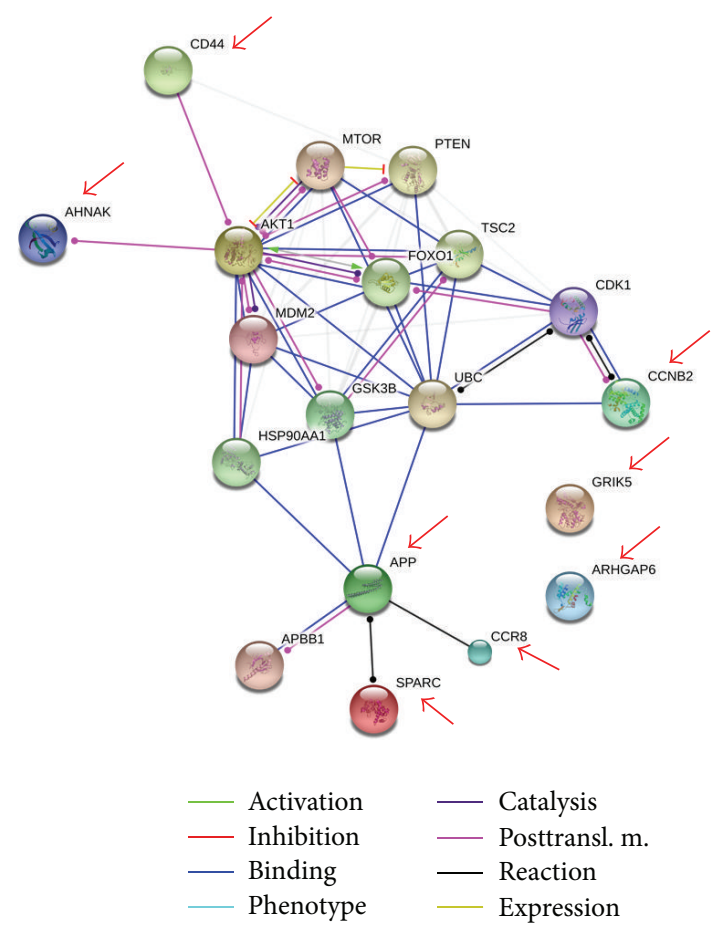

FIGURE 5: The protein-protein interaction pathways were performed. Proteins identified in this study were marked by arrows. The CD44 and APP can turn on the PI3K/AKT/mTOR pathway, which is responsible for the proliferation and is required for survival of the majority of cells.

was identified by three or more unique peptides, no visual assessment of spectra was conducted and the protein was considered to be present in the sample.

In this study, more than one hundred proteins were identified and most of these were identified at the minimal confidence level, which was only one unique peptide sequence matched. Experimental results reported a 


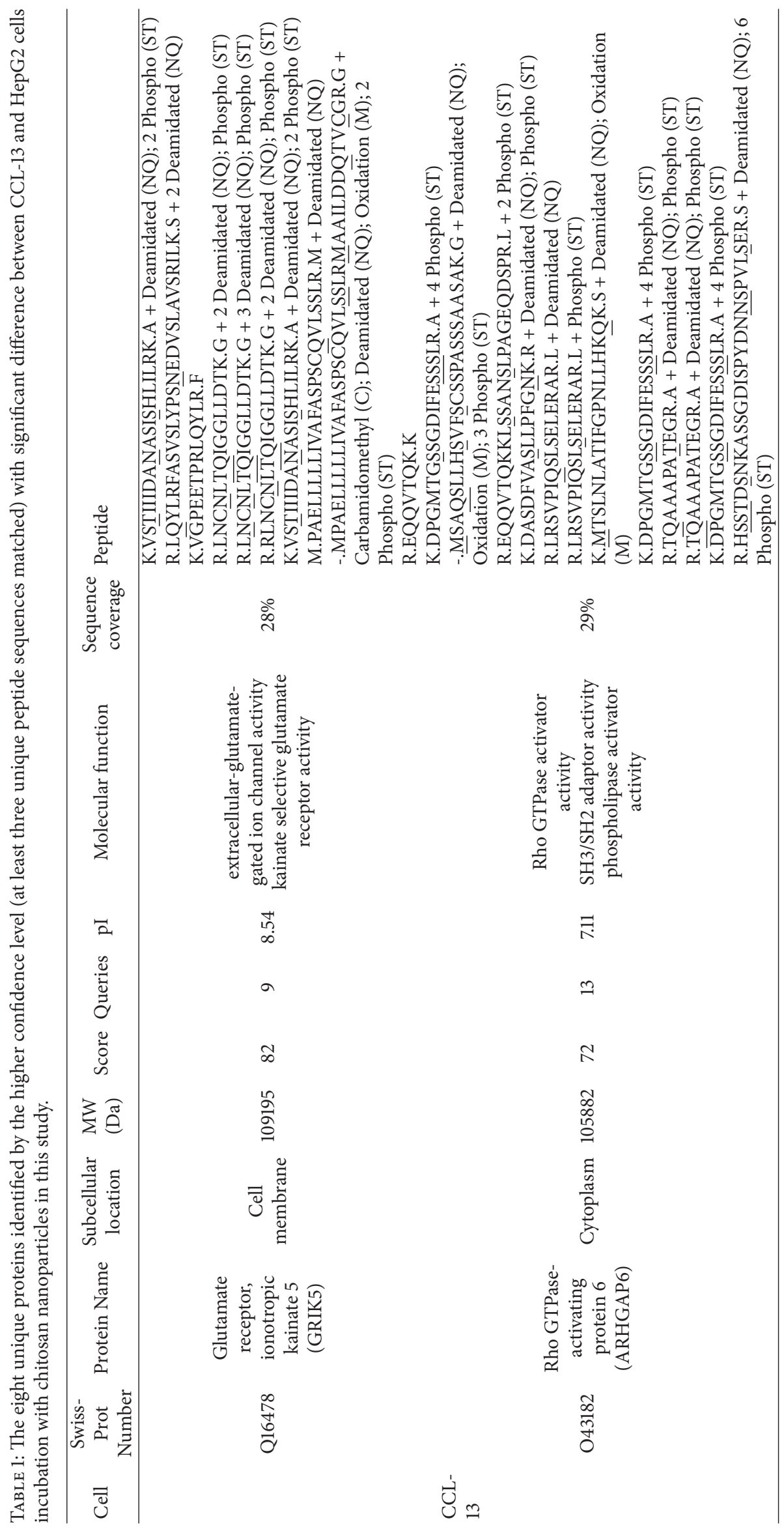




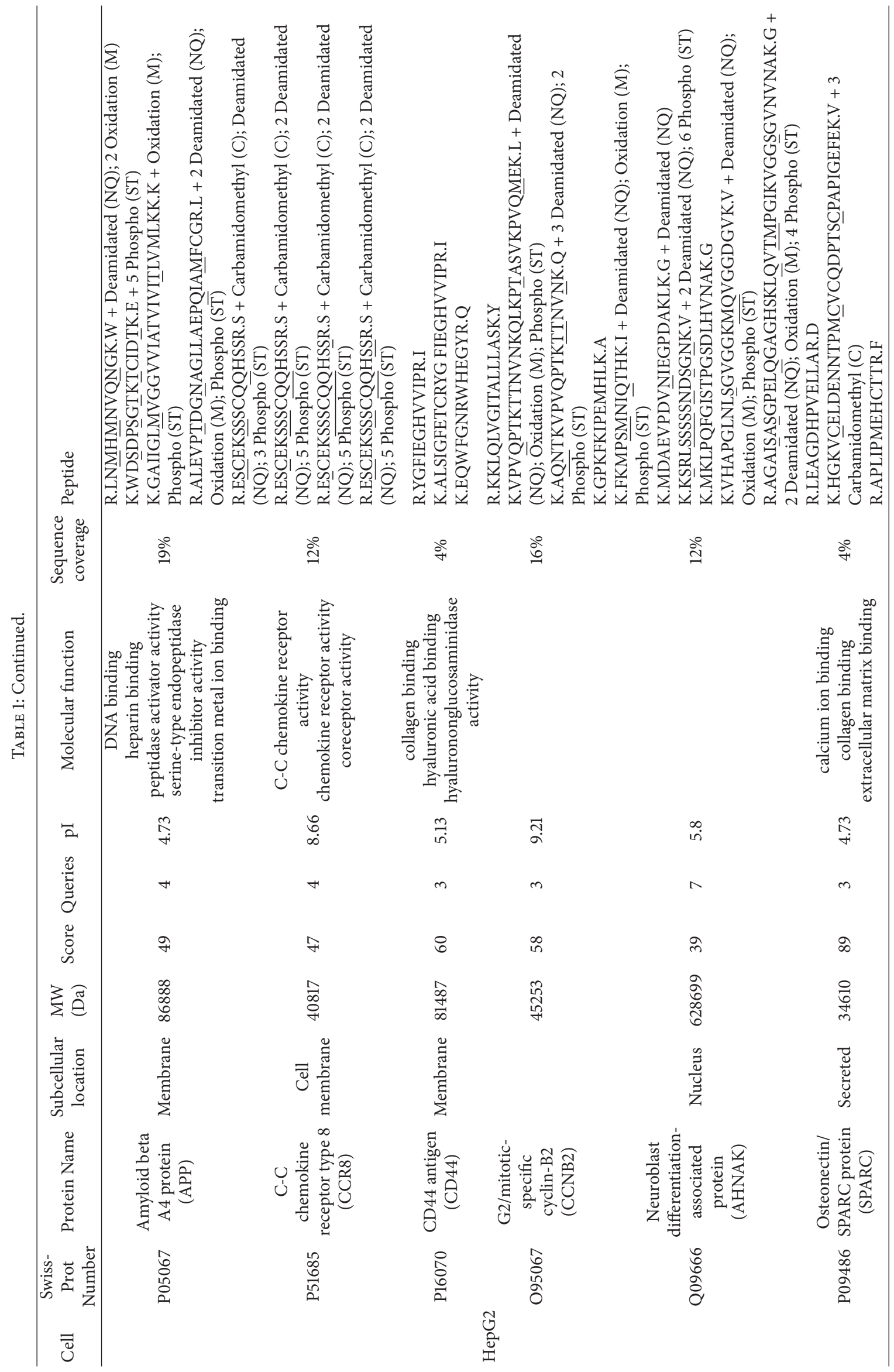


total of eight protein identifications with higher confidence levels (at least three unique peptide sequences matched) and exhibited significant differences between the chitosan nanoparticle treated and nontreated cells. Those cell lysate proteins were involved in cell growth, differentiation, division, cycle regulation (6 in HepG2 cells and 2 in CCL-13 cells).

A summary of the protein identifications achieved is shown in Table 1. For the eight protein identifications, six proteins were positively identified as Amyloid beta A4 protein (APP, P05067), C-C chemokine receptor type 8 (CCR8, P51685), CD44 antigen (CD44, P16070), G2/mitotic-specific cyclin-B2 (CCNB2, O95067), Neuroblast differentiation-associated protein (AHNAK, Q09666), and Osteonectin/SPARC protein (SPARC, P09486) in HepG2 cell lysate. Two proteins, Glutamate receptor ionotropic, kainate 5 (GRIK5, Q16478), and Rho GTPase-activating protein 6 (ARHGAP6, O43182) were found in the CCL-13 cell lysate. The protein-protein interaction pathways were performed by String 9.1 Web software. Eight proteins identified in this study were marked by red arrows (Figure 5).

GRIK5 and ARHGAP6 were not detected in the HepG2 cells but in CCL-13 cells. GRIK5 gene belongs to the kainate family of glutamate receptor in the cerebellum and the suprachiasmatic nuclei (SCN) of the hypothalamus, which is composed of four subunits and function as ligandactivated ion channels [26]. It is the predominant excitatory neurotransmitter receptor in the brain of mammalian and is involved in the neurophysiologic processes, such as the transmission of light information from the retina to the hypothalamus.

ARHGAP6 is a regulatory protein which can bind to activated $G$ proteins and stimulate their GTPase activity. Regulation of $\mathrm{G}$ proteins is important because these proteins are involved in a variety of important cellular processes. It may result in transduction of signaling from the G proteincoupled receptor for a variety of signaling processes like hormonal signaling and involve in processes like cellular trafficking and cell cycling [27]. In this study, GRIK5 and ARHGAP6 were identified in the CCL-13 cells after chitosan nanoparticle treatment. However, the analyses of protein functions and protein-protein interaction pathways did not show the serious effect in CCL-13 cells.

In this study, the main finding of chitosan nanoparticle treated cells is the chitosan nanoparticles enhancing the $\mathrm{PI} 3 \mathrm{~K} / \mathrm{AKT} 1 / \mathrm{mTOR}$ pathway in Hep-G2 cells which may result in tumor metastasis. There were six proteins identified in HepG-2 cell lysate samples, which may relate to metastasis and cell proliferation. Using the protein-protein interaction pathway analysis, those six proteins were related to the PI3K/AKT1/mTOR pathway (Figure 5). The CD44 can turn on the PI3K/AKT1/mTOR pathway, which is responsible for the proliferation and is required for survival of the majority of cells. The PI3K/AKT1/mTOR pathway can also be turned on by GSK3B when GSK3B is bound with APP and SPARC.

The mammalian target of rapamycin pathway (mTOR pathway, also known as FRAP, RAFT1, and RAP1 pathway) has been identified as a key kinase acting downstream of the activation of phosphoinositide-3-kinase (PI3K) [28]. The $\mathrm{PI} 3 \mathrm{~K} / \mathrm{AKT} 1 / \mathrm{mTOR}$ pathway is responsible for the proliferation and is required for survival of the majority of cancer cells. The hypothesis of the mTOR pathway is acting as a master switch of cellular catabolism and anabolism, thereby determining whether cells grow and proliferate of tumor cells $[29,30]$. Activation of PI3K/AKT1/mTOR signaling through mutation of pathway components as well as through activation of upstream signaling molecules occurs in a majority of cancer cells contributing to deregulation of proliferation, resistance to apoptosis, and changes in metabolism characteristic of transforming cells [31].

\section{Conclusion}

As previous reports have indicated that the chitosan nanoparticle was nontoxic for cell lines and appropriate as a drug carrier in micro capsule. In this study, the experimental results showed that it is a dose dependent manner to enhance cell growth. However, according to the proteomics analysis, chitosan nanoparticle induced the liver cancer cell to secret several proteins which may active or enhance $\mathrm{PI} 3 \mathrm{~K} / \mathrm{AKT} 1 / \mathrm{mTOR}$ pathway. The PI3K/AKT1/mTOR pathway was related to the cancer call metastasis and proliferation. Although there are no direct evidences to prove the relevance between chitosan nanoparticle and PI3K/AKT1/mTOR pathway in our study, it is still worth to be considered, especially as an anticancer drug delivery system.

\section{Conflict of Interests}

The authors declare that there is no conflict of interests regarding the publication of this paper.

\section{Authors' Contribution}

Ming-Hui Yang and Shyng-Shiou Yuan contributed equally to this work as first authors.

\section{Acknowledgments}

The authors thank the Center of Excellence for Environmental Medicine, Kaohsiung Medical University for the assistance in protein identification. This work was supported by research Grant nos. NSC-102-3114-Y-492-076-023, NSC-1012221-E-010-013-MY2, NSC-100-2320-B-037-007-MY3, and NSC-099-2811-E-224-002 from the National Science Council, MOHW103-TD-B-111-05 from Ministry of Health and Welfare, and NSYSUKMU 102-P006 from NSYSU-KMU Joint Research Project, Taiwan.

\section{References}

[1] Q. Li, E. T. Dunn, E. W. Grandmaison, and M. F. A. Goosen, "Applications and properties of chitosan," Journal of Bioactive and Compatible Polymers, vol. 7, no. 4, pp. 370-397, 1992. 
[2] L. Shang and G. U. Nienhaus, "Small fluorescent nanoparticles at the nano-bio interface," Materials Today, vol. 16, no. 3, pp. 58-66, 2013.

[3] P. Orlowski, M. Krzyzowska, R. Zdanowski et al., "Assessment of in vitro cellular responses of monocytes and keratinocytes to tannic acid modified silver nanoparticles," Toxicology in Vitro, vol. 27, no. 6, pp. 1798-1808, 2013.

[4] C. Zanette, M. Pelin, M. Crosera et al., "Silver nanoparticles exert a long-lasting antiproliferative effect on human keratinocyte HaCaT cell line," Toxicology in Vitro, vol. 25, no. 5, pp. 1053-1060, 2011.

[5] S. J. Soenen, B. Manshian, J. M. Montenegro et al., "Cytotoxic effects of gold nanoparticles: a multiparametric study," ACS Nano, vol. 6, no. 7, pp. 5767-5783, 2012.

[6] B. D. Holt, K. N. Dahl, and M. F. Islam, "Cells take up and recover from protein-stabilized single-wall carbon nanotubes with two distinct rates," ACS Nano, vol. 6, no. 4, pp. 3481-3490, 2012.

[7] S. Hirano and Y. Noishiki, "The blood compatibility of chitosan and N-acylchitosans," Journal of Biomedical Materials Research, vol. 19, no. 4, pp. 413-417, 1985.

[8] K. Y. Lee and D. J. Mooney, "Alginate: properties and biomedical applications," Progress in Polymer Science, vol. 37, no. 1, pp. 106126, 2012.

[9] M. R. Kasaai, J. Arul, and G. Charlet, "Fragmentation of chitosan by acids," The Scientific World Journal, vol. 2013, Article ID 508540, 11 pages, 2013.

[10] K. S. V. Krishna Rao, B. Vijaya Kumar Naidu, M. C. S. Subha, M. Sairam, and T. M. Aminabhavi, "Novel chitosan-based pHsensitive interpenetrating network microgels for the controlled release of cefadroxil," Carbohydrate Polymers, vol. 66, no. 3, pp. 333-344, 2006.

[11] M. Rani, A. Agarwal, and Y. Negi, "Characterization and biodegradation studies for interpenetrating polymeric network (IPN) of chitosan-amino acid beads," Journal of Biomaterials and Nanobiotechnology, vol. 2, no. 1, pp. 71-84, 2011.

[12] M. E. I. Badawy and E. I. Rabea, "A biopolymer chitosan and its derivatives as promising antimicrobial agents against plant pathogens and their applications in crop protection," International Journal of Carbohydrate Chemistry, vol. 2011, Article ID 460381, 29 pages, 2011.

[13] J.-I. Murata, Y. Ohya, and T. Ouchi, "Possibility of application of quaternary chitosan having pendant galactose residues as gene delivery tool," Carbohydrate Polymers, vol. 29, no. 1, pp. 69-74, 1996.

[14] P. Kandra, M. M. Challa, and H. K. Jyothi, "Efficient use of shrimp waste: present and future trends," Applied Microbiology and Biotechnology, vol. 93, no. 1, pp. 17-29, 2012.

[15] S. Minami, H. Suzuki, Y. Okamoto, T. Fujinaga, and Y. Shigemasa, "Chitin and chitosan activate complement via the alternative pathway," Carbohydrate Polymers, vol. 36, no. 2-3, pp. 151-155, 1998.

[16] J. Zhang, W. Xia, P. Liu et al., "Chitosan modification and pharmaceutical/biomedical applications," Marine Drugs, vol. 8, no. 7, pp. 1962-1987, 2010.

[17] F. Talaei, M. Azhdarzadeh, H. Hashemi Nasel et al., "Core shell methyl methacrylate chitosan nanoparticles: in vitro mucoadhesion and complement activation," DARU, Journal of Pharmaceutical Sciences, vol. 19, no. 4, pp. 257-265, 2011.

[18] J. Venkatesan and S.-K. Kim, "Chitosan composites for bone tissue engineering: an overview," Marine Drugs, vol. 8, no. 8, pp. 2252-2266, 2010.
[19] P. Calvo, C. Remuñan-López, J. L. Vila-Jato, and M. J. Alonso, "Chitosan and chitosan/ethylene oxide-propylene oxide block copolymer nanoparticles as novel carriers for proteins and vaccines," Pharmaceutical Research, vol. 14, no. 10, pp. 1431-1436, 1997.

[20] I. M. Van der Lubben, J. C. Verhoef, G. Borchard, and H. E. Junginger, "Chitosan for mucosal vaccination," Advanced Drug Delivery Reviews, vol. 52, no. 2, pp. 139-144, 2001.

[21] T. López-León, E. L. S. Carvalho, B. Seijo, J. L. Ortega-Vinuesa, and D. Bastos-González, "Physicochemical characterization of chitosan nanoparticles: electrokinetic and stability behavior," Journal of Colloid and Interface Science, vol. 283, no. 2, pp. 344351, 2005.

[22] Y. Guichard, J. Schmit, C. Darne et al., "Cytotoxicity and genotoxicity of nanosized and microsized titanium dioxide and iron oxide particles in Syrian hamster embryo cells," The Annals of Occupational Hygiene, vol. 56, no. 5, pp. 631-644, 2012.

[23] J. C. K. Lai, M. B. Lai, S. Jandhyam et al., "Exposure to titanium dioxide and other metallic oxide nanoparticles induces cytotoxicity on human neural cells and fibroblasts," International Journal of Nanomedicine, vol. 3, no. 4, pp. 533-545, 2008.

[24] R. Liu, R. Rallo, S. George et al., "Classification NanoSAR development for cytotoxicity of metal oxide nanoparticles," Small, vol. 7, no. 8, pp. 1118-1126, 2011.

[25] J. D. Jaffe, H. C. Berg, and G. M. Church, "Proteogenomic mapping as a complementary method to perform genome annotation," Proteomics, vol. 4, no. 1, pp. 59-77, 2004.

[26] P. E. Schauwecker, "Differences in ionotropic glutamate receptor subunit expression are not responsible for strain-dependent susceptibility to excitotoxin-induced injury," Molecular Brain Research, vol. 112, no. 1-2, pp. 70-81, 2003.

[27] A. J. Kimple, M. Soundararajan, S. Q. Hutsell et al., "Structural determinants of G-protein $\alpha$ subunit selectivity by regulator of G-protein signaling 2 (RGS2)," Journal of Biological Chemistry, vol. 284, no. 29, pp. 19402-19411, 2009.

[28] S. Faivre, G. Kroemer, and E. Raymond, "Current development of mTOR inhibitors as anticancer agents," Nature Reviews Drug Discovery, vol. 5, no. 8, pp. 671-688, 2006.

[29] N. Khan, F. Afaq, F. H. Khusro, V. Mustafa Adhami, Y. Suh, and H. Mukhtar, "Dual inhibition of phosphatidylinositol 3kinase/Akt and mammalian target of rapamycin signaling in human nonsmall cell lung cancer cells by a dietary flavonoid fisetin," International Journal of Cancer, vol. 130, no. 7, pp. 16951705, 2012.

[30] K. E. O’Reilly, F. Rojo, Q.-B. She et al., "mTOR inhibition induces upstream receptor tyrosine kinase signaling and activates Akt," Cancer Research, vol. 66, no. 3, pp. 1500-1508, 2006.

[31] H.-Y. Zhou and S.-L. Huang, "Current development of the second generation of mTOR inhibitors as anticancer agents," Chinese Journal of Cancer, vol. 31, no. 1, pp. 8-18, 2012. 

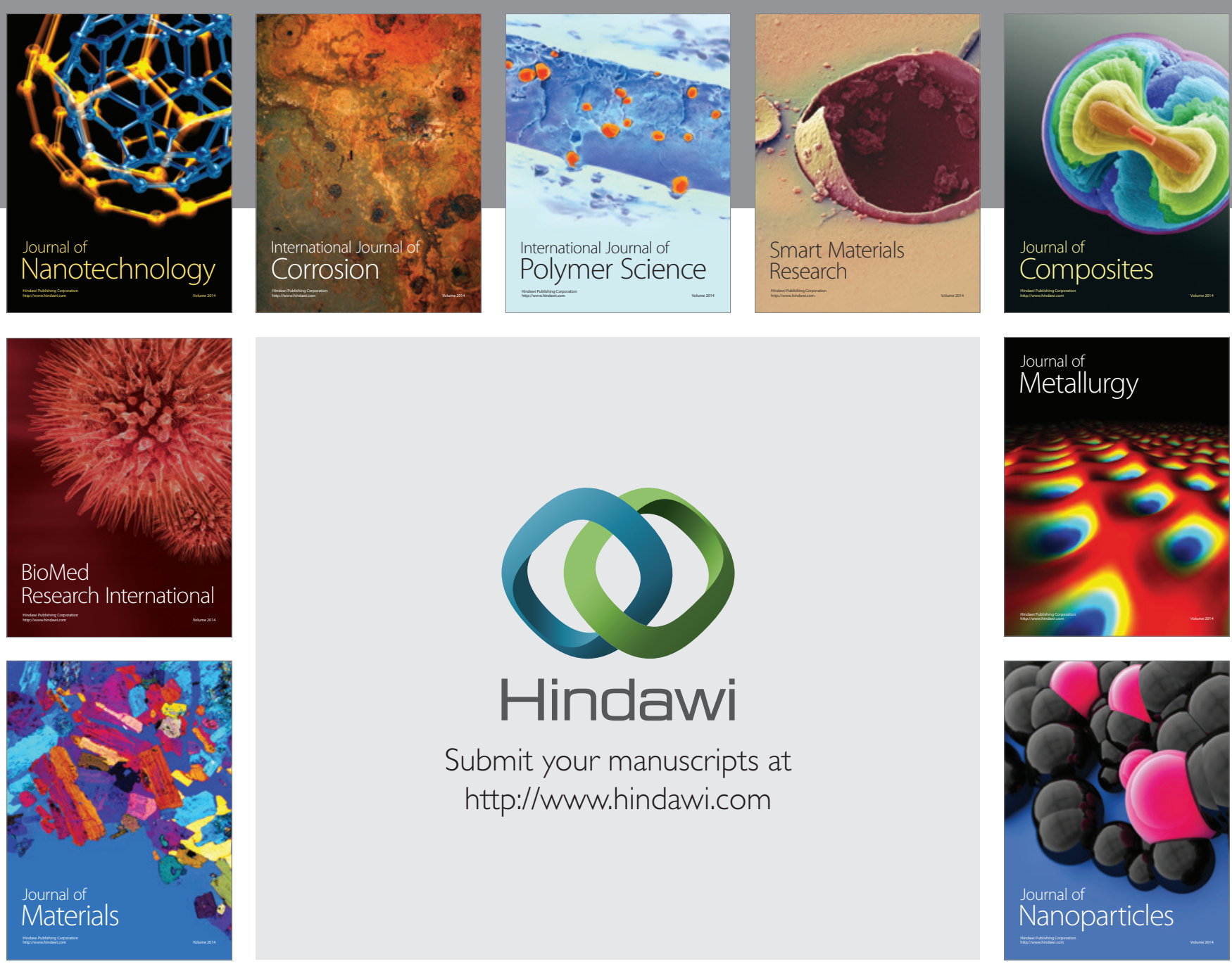

Submit your manuscripts at http://www.hindawi.com
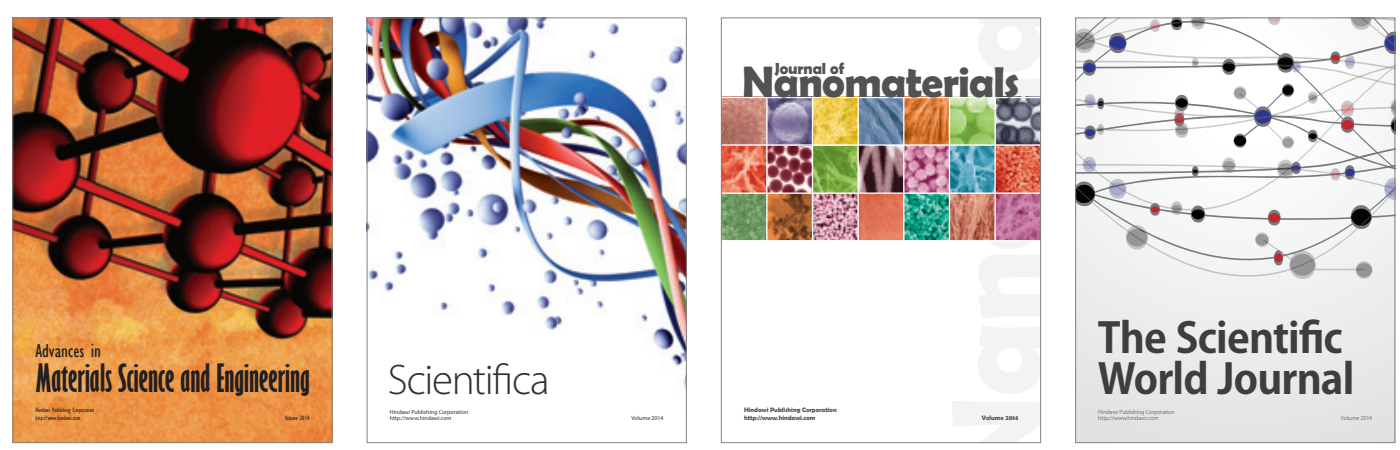

\section{The Scientific World Journal}
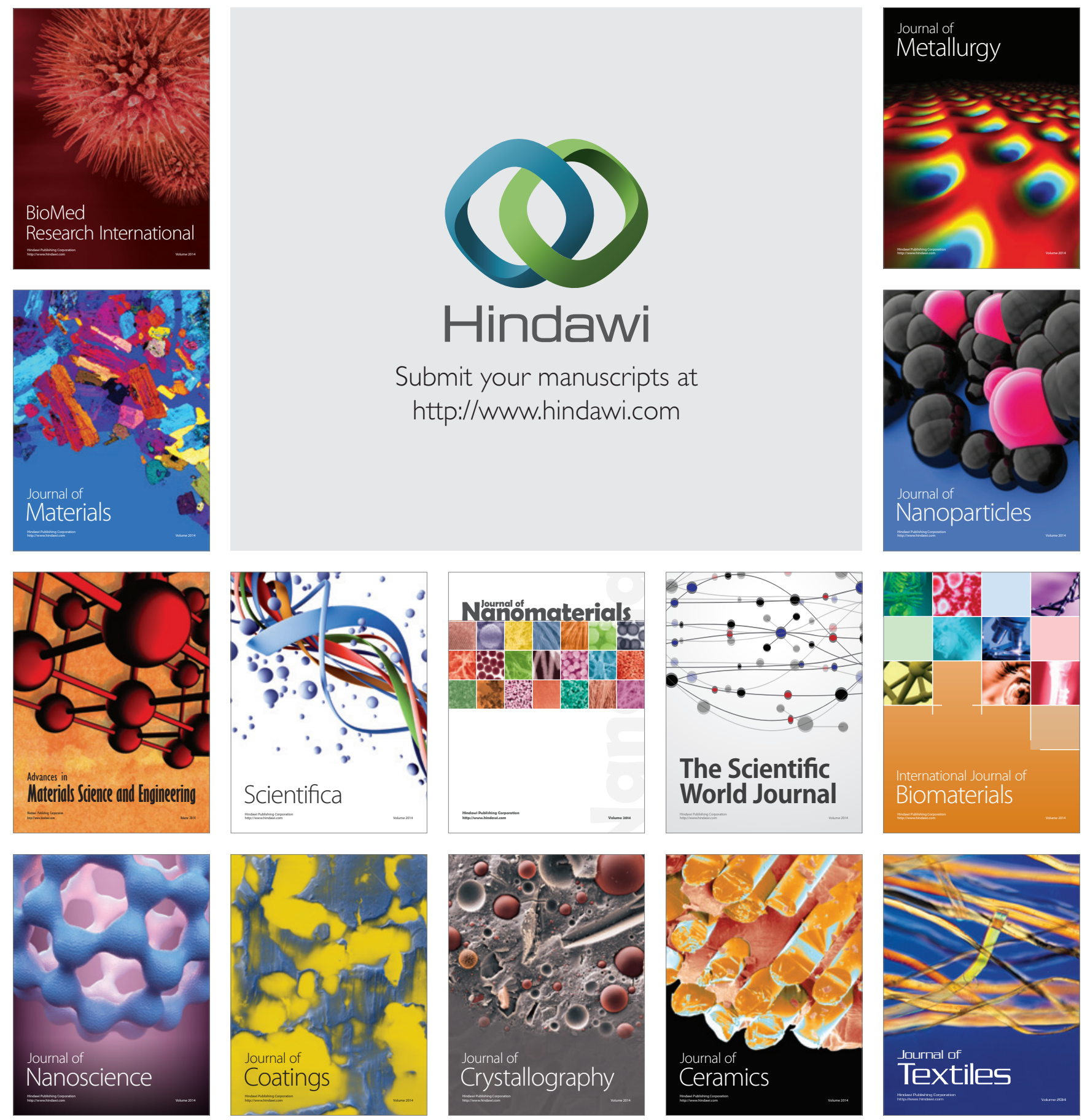